\title{
Adverse early life experiences are associated with changes in pressure and cold pain sensitivity in young adults
}

\author{
Line Caes $^{1 \wedge}$, Michelle Roche ${ }^{2 \wedge}$ \\ ${ }^{1}$ Department of Psychology, Faculty of Natural Sciences, University of Stirling, Scotland, UK; ${ }^{2}$ Physiology, School of Medicine, Galway \\ Neuroscience Centre and Centre for Pain Research, National University of Ireland Galway, Galway, Ireland \\ Correspondence to: Michelle Roche. Physiology, School of Medicine, National University of Ireland Galway, University Road, Galway, Ireland. \\ Email: Michelle.roche@nuigalway.ie. \\ Provenance and Peer Review: This article was commissioned by the editorial office, Annals of Palliative Medicine. The article did not undergo external \\ peer review. \\ Comment on: Waller R, Smith AJ, O'Sullivan PB, et al. The association of early life stressors with pain sensitivity and pain experience at 22 years. Pain \\ 2020;161:220-9.
}

Submitted Apr 09, 2020. Accepted for publication May 09, 2020.

doi: 10.21037/apm-20-914

View this article at: http://dx.doi.org/10.21037/apm-20-914

Over the past two decades, understanding on the importance and impact of early life events on health and well-being in later life has expanded considerably. Accordingly, childhood stress and trauma during this critical period has been demonstrated to elicit profound changes physiological functioning, which in turn is associated with an increased risk of developing conditions such as chronic pain. Preclinical and animal studies have provided evidence for changes in several neurobiological substrates including the stress hypothalamic-pituitary-adrenal axis, immune system and epigenetic mechanisms, in the association between early life stress and altered pain processing in later life $(1,2)$. However, clinical data is limited and often conflicted on whether childhood stress and trauma results in increased $(3,4)$ or decreased $(4-6)$ pain sensitivity or experience. The Western Australian pregnancy cohort (Raine) Study commenced in 1989 and has provided longitudinal physiological, psychological and socioeconomic data on a large cohort of parents and offspring over the past 30 years. A recent study by Waller and colleagues published in Pain, utilized data from the Raine study to assess the association between a wide range of early life stressors, pressure and cold sensitivity and pain experience in young adults (7). The authors are to be commended for their rigorous analyses of this large cohort data resulting in robust data which demonstrates that adverse early life experiences are associated with changes in pressure and cold pain sensitivity at 22 years. These data provide further support that early life experiences are associated with altered long-term nociceptive processing. Given the lack of longitudinal studies in this area and the social and economic burden of treating acute and chronic pain, this study provides robust evidence on early life stress as a risk factor for altered pain processing thus providing valuable insight into possible biomarkers and novel treatment strategies for such conditions.

Increasing evidence supports a significant association between early life stress and adversity with altered pain responding and an increased incidence of chronic pain in later life $(1,6,8-10)$. However, many of the studies examining the association between early life stress and pain in later life have been retrospective studies or specifically examine the impact of neonatal pain. The study by Waller and colleagues (7) utilised the Raine study which enabled the authors to use a comprehensive longitudinal data set to examine a wide range of early life experiences in a large nonclinical cohort. The use of large cohort studies is a strong methodology for longitudinal studies, given the

^ORCID: Line Caes: 0000-0001-7355-0706; Michelle Roche: 0000-0002-7242-9397. 
large sample size and standardised assessment procedures. However, drawbacks of a cohort methodology include the potential for minimal input from the researchers on the exact measures being taken at each and every time point. Nevertheless, the authors were able to included standardised, best practice cold pain and pressure pain threshold assessment at 22 years of age. In addition, the authors had access to excellent records on early life stressful events both antenatal and over the first 3 years of life. These stressful events included questions such as problems with pregnancy, death of a close, relative, death of a friend, marital problems, separation or divorce, problems with children, involuntary loss of own job, involuntary loss of partners job, financial hardship, residential move, and "other" stressful events. Although the authors admit that data was missing from most variables which could potentially give rise to biased results, this was accounted for by adopting a robust statistical analysis strategy, such as the inclusion of multiple imputation data sets and bootstrapped backwards stepwise selection procedure. Consequently, the resulting outcomes and conclusions are robust and not inflated by a large sample size or biased due to a potentially large amount and various patterns of missing data. Overall the findings suggest divergent effects of early life stress on pressure and cold pain sensitivity, but not experience, in young adults.

Specifically, the research revealed that (I) more problematic behaviour at age 2 years was associated with less pressure pain sensitivity, but not cold sensitivity or pain experience, at 22 years; (II) poorer family functioning increased the odds for having high cold pain sensitivity at 22 years, and those reporting a moderate/high pain experience at 22 years; poor family functioning further increased the odds of higher cold pain sensitivity.

Divergent effects of early life stress on responses to noxious stimuli is not unusual with several studies having reported both increases and decreases in thresholds. As the authors suggest, these differential effects are likely due to different neuronal networks transmitting and modulating these stimuli. Indeed, these divergent findings for pressure and cold pain further add to our knowledge that different experimental pain induction methodologies induce different sensory and affective qualities of pain stimulations (11). However, to date, little is known about how these pain experiences induced by experimental induction techniques match exactly onto real-life pain experiences (12). What is more consistent is the association between early life adversity and increased incidence of chronic pain in later life. Although the current study included chronicity in its pain assessments at age 22 years, this was combined with intensity and number of pain areas in order to group individuals into low, medium or high pain groups. As such, with the current data it is not possible to delineate if there was a specific contribution of early life stress to the development of chronic pain in this cohort. For future studies it would be of interest to include separate indices of pain intensity, interference and chronicity to gain a comprehensive understanding of the potentially diverse impact of early life stressors on these different aspects of later life pain experiences.

The moderating relationship of early life family functioning on cold pain sensitivity further adds to the mostly cross-sectional, evidence that poor family functioning plays a role in understanding chronic pain experiences; especially disability due to pain (13). This is however, to our knowledge, the first study to highlight that poor family functioning is a potential early life risk factor for the development of heightened pain sensitivity, which could play a role in developing chronic pain later in life. Future studies prospectively exploring the association between family functioning, pain sensitivity and the development of chronic pain across the life span are warranted to shed more clarity on these complex interrelations and allow the identifications of early life risk factors for chronic pain development.

Interestingly, while females were found to exhibit higher pressure and cold pain sensitivity, the relationship with problematic behaviour and poorer family functioning on these parameters was independent of the participant's sex (7). Sex differences are widely acknowledged, with females demonstrating increased sensitivity to noxious stimuli and incidence of chronic pain. Previous evidence has shown that females with a history of sexual abuse exhibit lower pressure pain thresholds (14), however whether similar effects occur in males was not examined in this study. While the Waller study highlights a lack of sex effect in their analysis, further studies examining if sex differences exist on the impact of early life experience and stress on pain responding in later life is warranted.

While the data presented has expanded our understanding on the association between early life stress and pain sensitivity, a number of outstanding questions and limitations should be noted. For example, although a wide array of early life events were recorded and included in the analysis, events such as previous pain experiences $(4,15)$ and childhood trauma (16), which have been demonstrated to impact on 
pain processing, were not captured. Although the current data set support a likely role for early life stress in mediating changes in nociceptive responding, it is impossible to account for all potential confounds that may occur alongside those early life events recorded or subsequent to this period which in turn may impact on nociceptive processing. Furthermore, such events occur against a backdrop of varying levels of inherited vulnerability and in various social and cultural contexts.

For instance, given the Western Australian pregnancy cohort (Raine) includes data collection on regular intervals during the participant's development, an important aspect missing from this study's analyses is the inclusion of more frequent assessments of pain and family functioning/ events throughout the child's development. Such recurring assessment would allow further insight into the complex associations between early life stressful events and later life pain experiences. As the authors highlight, adolescence is a critical period of development associated with changes in social environment (i.e., relying more on peers than their parents) and gaining independence. Furthermore, an increase in the prevalence of chronic pain is apparent from adolescence onwards (17). However, what happens during adolescence in terms of their social environment and pain experiences is absent from this study but could potentially have a substantial moderating influence on the extent early life events and family circumstances impact on their pain experiences in young adulthood. For example, evidence indicates that having a reliable and trustworthy person to connect with (e.g., teacher or a peer) during adolescence can buffer the impact of negative family influences (18). However, this evidence is limited to assessment of stressful events taking place during adolescence, thereby preventing conclusions on how a supportive social environment outside of the family home during adolescence could buffer against the impact of early life stressful experiences.

In conclusion, the study by Waller and colleagues (in 2020) reveals the importance of early life stressors on pain sensitivity in young adults using an impressive longitudinal set-up and rigorous data analytical techniques. We anticipate that this data will encourage and stimulate more prospective and robust research designs in the future, thereby furthering our understanding of the impact of early life events on pain experiences throughout the life span. In such future research endeavors, it will be crucial to address the limitations of the Waller and colleagues (in 2020) study, such as the inclusion of independent measures for pain intensity, interference and chronicity as well as the inclusion of multiple assessment points of life events and pain experiences across the lifespan. Furthermore, the divergent findings for the impact of early life experiences depending on the adopted pain inducing modality (i.e., pressure and cold pain) and how this matches onto potential divergent impacts on different real life pain experiences warrants further exploration.

\section{Acknowledgments}

Funding: None.

\section{Footnote}

Conflicts of Interest: Both authors have completed the ICMJE uniform disclosure form (available at http://dx.doi. org/10.21037/apm-20-914). LC reports grants from Royal Society of Edinburgh (RSE), grants from National Institute for Health Research (NIHR), grants from British Psychological Society (BPS), outside the submitted work. MR reports grants from Alkermes Inc., grants from Science Foundation Ireland, outside the submitted work.

Ethical Statement: The authors are accountable for all aspects of the work in ensuring that questions related to the accuracy or integrity of any part of the work are appropriately investigated and resolved.

Open Access Statement: This is an Open Access article distributed in accordance with the Creative Commons Attribution-NonCommercial-NoDerivs 4.0 International License (CC BY-NC-ND 4.0), which permits the noncommercial replication and distribution of the article with the strict proviso that no changes or edits are made and the original work is properly cited (including links to both the formal publication through the relevant DOI and the license). See: https://creativecommons.org/licenses/by-nc-nd/4.0/.

\section{References}

1. Burke NN, Finn DP, McGuire BE, et al. Psychological stress in early life as a predisposing factor for the development of chronic pain: Clinical and preclinical evidence and neurobiological mechanisms. J Neurosci Res 2017;95:1257-70.

2. Agorastos A, Pervanidou P, Chrousos GP, et al. Developmental Trajectories of Early Life Stress and Trauma: A Narrative Review on Neurobiological Aspects 
Beyond Stress System Dysregulation. Front Psychiatry 2019;10:118.

3. Sherman AL, Morris MC, Bruehl S, et al. Heightened Temporal Summation of Pain in Patients with Functional Gastrointestinal Disorders and History of Trauma. Ann Behav Med 2015;49:785-92.

4. Walker SM, Melbourne A, O'Reilly H, et al. Somatosensory function and pain in extremely preterm young adults from the UK EPICure cohort: sex-dependent differences and impact of neonatal surgery. $\mathrm{Br} \mathrm{J}$ Anaesth 2018;121:623-35.

5. Pieritz K, Rief W, Euteneuer F. Childhood adversities and laboratory pain perception. Neuropsychiatr Dis Treat 2015;11:2109-16.

6. Fillingim RB, Edwards RR. Is self-reported childhood abuse history associated with pain perception among healthy young women and men? Clin J Pain 2005;21:387-97.

7. Waller R, Smith AJ, O'Sullivan PB, et al. The association of early life stressors with pain sensitivity and pain experience at 22 years. Pain 2020;161:220-9.

8. Low LA, Schweinhardt P. Early life adversity as a risk factor for fibromyalgia in later life. Pain Res Treat 2012;2012:140832.

9. Barreau F, Ferrier L, Fioramonti J, et al. New insights in the etiology and pathophysiology of irritable bowel syndrome: contribution of neonatal stress models. Pediatr Res 2007;62:240-5.

10. Afari N, Ahumada SM, Wright LJ, et al. Psychological trauma and functional somatic syndromes: a systematic review and meta-analysis. Psychosom Med 2014;76:2-11.

Cite this article as: Caes L, Roche M. Adverse early life experiences are associated with changes in pressure and cold pain sensitivity in young adults. Ann Palliat Med 2020;9(4):1366-1369. doi: 10.21037/apm-20-914
11. Neziri AY, Curatolo M, Nuesch E, et al. Factor analysis of responses to thermal, electrical, and mechanical painful stimuli supports the importance of multi-modal pain assessment. Pain 2011;152:1146-55.

12. Birnie KA, Caes L, Wilson AC, et al. A practical guide and perspectives on the use of experimental pain modalities with children and adolescents. Pain management 2014;4:97-111.

13. Lewandowski AS, Palermo TM, Stinson J, et al. Systematic review of family functioning in families of children and adolescents with chronic pain. J Pain 2010;11:1027-38.

14. Scarinci IC, McDonald-Haile J, Bradley LA, et al. Altered pain perception and psychosocial features among women with gastrointestinal disorders and history of abuse: a preliminary model. Am J Med 1994;97:108-18.

15. Walker SM, Franck LS, Fitzgerald M, et al. Longterm impact of neonatal intensive care and surgery on somatosensory perception in children born extremely preterm. Pain 2009;141:79-87.

16. Davis DA, Luecken LJ, Zautra AJ. Are reports of childhood abuse related to the experience of chronic pain in adulthood? A meta-analytic review of the literature. Clin J Pain 2005;21:398-405.

17. King S, Chambers CT, Huguet A, et al. The epidemiology of chronic pain in children and adolescents revisited: a systematic review. Pain 2011;152:2729-38.

18. Vervoort T, Logan DE, Goubert L, et al. Severity of pediatric pain in relation to school-related functioning and teacher support: an epidemiological study among schoolaged children and adolescents. Pain 2014;155:1118-27. 